PROCEEDINGS OF THE

AMERICAN MATHEMATICAL SOCIETY

Volume 127, Number 8, Pages 2489-2496

S 0002-9939(99)04781-4

Article electronically published on April 15, 1999

\title{
N-COMPACTNESS AND AUTOMATIC CONTINUITY IN ULTRAMETRIC SPACES OF BOUNDED CONTINUOUS FUNCTIONS
}

\author{
JESÚS ARAUJO
}

(Communicated by Alan Dow)

\begin{abstract}
In this paper (weakly) separating maps between spaces of bounded continuous functions over a nonarchimedean field $\mathbb{K}$ are studied. It is proven that the behaviour of these maps when $\mathbb{K}$ is not locally compact is very different from the case of real- or complex-valued functions: in general, for $\mathbb{N}$ compact spaces $X$ and $Y$, the existence of a (weakly) separating additive map $T: C^{*}(X) \rightarrow C^{*}(Y)$ implies that $X$ and $Y$ are homeomorphic, whereas when dealing with real-valued functions, this result is in general false, and we can just deduce the existence of a homeomorphism between the Stone-Čech compactifications of $X$ and $Y$. Finally, we also describe the general form of bijective weakly separating linear maps and deduce some automatic continuity results.
\end{abstract}

\section{INTRODUCTION}

It is well-known that the existence of a ring isomorphism between the spaces $C(X)$ and $C(Y)$ of real-valued continuous functions on the real compact spaces $X$ and $Y$ always implies the existence of a homeomorphism between $X$ and $Y$ (see for instance [GJ, Theorem 8.3]). In [ABN1] a similar result was given for biseparating additive maps (see definition below) instead of ring isomorphisms. Also, when working with biseparating maps between spaces of continuous functions over a nonarchimedean valued field, roughly the result remains true, although in this case we do not assume the spaces $X$ and $Y$ to be real-compact but $\mathbb{N}$-compact (see $[\mathrm{ABN} 2])$.

As for the case when we study the ring isomorphisms or biseparating additive maps between the spaces $C^{*}(X)$ and $C^{*}(Y)$ of real-valued bounded continuous functions on $X$ and $Y$, we conclude that the Stone-Čech compactifications of $X$ and $Y$ are homeomorphic, since every continuous function can be extended to a continuous function in the Stone-Čech compactification. Likewise, a similar result can be obtained when we study this kind of maps between spaces of bounded continuous functions taking values in a locally compact nonarchimedean field: in this case we conclude that the Banaschewski compactifications of $X$ and $Y$ are homeomorphic.

Received by the editors July 20, 1997 and, in revised form, November 6, 1997.

1991 Mathematics Subject Classification. Primary 54C40, 46S10.

Key words and phrases. $\mathbb{N}$-compact, weakly separating map, nonarchimedean field.

Research supported in part by the Spanish Dirección General de Investigación Científica y Técnica (DGICYT, PB95-0582). 
But as we will see in this paper, all of these results depend strongly on the local compactness of the ground field. For instance, for $\mathbb{N}$-compact spaces $X$ and $Y$, when $\mathbb{K}$ is not locally compact and has characteristic zero, the existence of a weakly biseparating and additive map $T: C^{*}(X) \rightarrow C^{*}(Y)$ (in particular, a ring isomorphism) implies that $X$ and $Y$ are homeomorphic (Theorem 2.8). Finally, we also give some results which allow us to describe the bijective weakly separating linear maps (Theorem 3.2). It is worth noting that, unlike in the case when $\mathbb{K}=$ $\mathbb{R}$, in our spaces no structure of lattice is available, and consequently techniques involving lattice structures cannot be carried out (see $[\mathrm{H}]$ and [GJ]).

Some related results (in the real or complex context) have also been given in, for instance, $[A B N 3]$ and $[\mathrm{J}]$, where linearity is regarded. As for the spaces of nonarchimedean bounded continuous functions, they have been studied, as Banach algebras, in $[\mathrm{vP}]$ and $[\mathrm{S}]$. Recently some other papers have also appeared linking the structure of spaces of continuous functions not only as rings but also as Banach spaces, for real and nonarchimedean fields as well (see for instance $[\mathrm{A}]$ and $[\mathrm{AF}]$ ), generalizing classical results in the context of the Banach-Stone theorem.

From now on $\mathbb{K}$ will be a commutative complete nonarchimedean valued field endowed with a nontrivial valuation. $X$ and $Y$ will be $\mathbb{N}$-compact spaces, and we will denote by $\beta_{0} X$ and $\beta_{0} Y$ their Banaschewski compactifications. If $U$ is a clopen (this is, open and closed) subset of $X, \xi_{U}$ will stand for the characteristic function on $U$, and if $a \in \mathbb{K}, \mathbf{a}:=a \xi_{X}$. As above, $C^{*}(X)$ and $C^{*}(Y)$ will be the spaces of $\mathbb{K}$-valued bounded continuous functions on $X$ and $Y$, respectively, endowed with the sup norm. For $f \in C^{*}(X)$ we will denote by $c(f)$ its cozero set $\{x \in X: f(x) \neq 0\}$, and by $f^{\beta_{0} X}$ its continuous extension from $\beta_{0} X$ into $\beta_{0} \mathbb{K}$. If $A \subset B, \operatorname{cl}_{B} A$ will be the closure of $A$ in $B$. Finally, for $T: C^{*}(X) \rightarrow C^{*}(Y)$, we define $Y_{0}:=\bigcup_{f \in C^{*}(X)} c(T f)$.

For basic facts on nonarchimedean fields and nonarchimedean Banach spaces, we refer to $[\mathrm{vR}]$.

\section{WEAKLY BISEPARATING MAPS}

Definition 2.1. A map $T: C^{*}(X) \rightarrow C^{*}(Y)$ is said to be separating if $(T f)(T g)=$ 0 whenever $f g=0$. It is said to be weakly separating if $(T f)(T g)=0$ whenever there exist disjoint clopen subsets $U$ and $V$ of $X$ containing $c(f)$ and $c(g)$, respectively. If $T$ is bijective, it is said to be (weakly) biseparating if both $T$ and $T^{-1}$ are (weakly) separating.

Definition 2.2. Suppose that $T$ is a weakly separating map from $C^{*}(X)$ into $C^{*}(Y)$. Given $y_{0} \in Y$, a point $x_{0} \in \beta_{0} X$ is said to be a support point of $y_{0}$ if for every neighborhood $U$ of $x_{0}$ in $\beta_{0} X$, there exists $f \in C^{*}(X)$ such that $c(f) \subset U$ and $(T f)\left(y_{0}\right) \neq 0$.

The existence and uniqueness of support points are given in the following theorem, which can be proved, with slight changes, as the corresponding one which appears in [BNT, p. 260].

Theorem 2.1. If $T: C^{*}(X) \rightarrow C^{*}(Y)$ is a weakly separating additive map, then for each $y \in Y_{0}$, there exists a unique support point in $\beta_{0} X$.

As a consequence of this result, we can define the map $h: Y_{0} \rightarrow \beta_{0} X$ sending each point of $Y_{0}$ into its support point. 
Lemma 2.2. Suppose that $T: C^{*}(X) \rightarrow C^{*}(Y)$ is a weakly separating additive map. If $y$ belongs to $Y_{0}$ and $f \in C^{*}(X)$ satisfies $(T f)(y) \neq 0$, then $h(y)$ belongs to $\operatorname{cl}_{\beta_{0} X} c(f)$.

Proof. Suppose that $h(y) \notin \operatorname{cl}_{\beta_{0} X} c(f)$. Then there exists a clopen neighborhood $U$ of $h(y)$ in $\beta_{0} X$ such that $U \cap \operatorname{cl}_{\beta_{0} X} c(f)=\emptyset$. On the other hand, there exists $g \in C^{*}(X)$ such that $c(g) \cap U \cap X=\emptyset$ and $(T g)(y) \neq 0$. This contradicts the fact that $T$ is weakly separating.

Corollary 2.3. Suppose that $T: C^{*}(X) \rightarrow C^{*}(Y)$ is an injective, weakly separating additive map. Then $h: Y_{0} \rightarrow \beta_{0} X$ is continuous and the image of $h$ is a dense subspace of $\beta_{0} X$.

Proof. First, we prove that $h$ is continuous. Take $y \in Y_{0}$ and a clopen neighborhood $U$ of $h(y)$ in $\beta_{0} X$. Suppose that $f \in C^{*}(X), f \neq 0$, satisfies $c(f) \subset U$ and $(T f)(y) \neq 0$. Then by Lemma $2.2, h(c(T f)) \subset U$, and consequently $h$ is continuous.

On the other hand, given a clopen nonempty subset $V$ of $\beta_{0} X, T \xi_{V \cap X}$ is not zero and $c\left(T \xi_{V \cap X}\right)$ is nonempty, since $T$ is injective. By Lemma 2.2, we conclude that $h\left(c\left(T \xi_{V \cap X}\right)\right) \subset V$ and that the image of $h$ is dense in $X$.

As a consequence we can extend $h$ to a continuous map from $\beta_{0} Y_{0}$ to $\beta_{0} X$. Also, if $T$ is injective, we deduce that its continuous extension to $\beta_{0} Y_{0}$ is surjective, since the range of $h$ is dense in $\beta_{0} X$. From now on we will also denote by $h$ this extension to $\beta_{0} Y_{0}$.

Lemma 2.4. Suppose that $T: C^{*}(X) \rightarrow C^{*}(Y)$ is a weakly separating additive map. If $U$ is a clopen subset of $\beta_{0} X$ and $f^{\beta_{0} X}(x)=0$ for every $x \in U$, then $(T f)^{\beta_{0} Y}(y)=0$ for every $y \in h^{-1}(U)$.

Proof. By Lemma 2.2, the result is clear for every $y \in Y_{0} \cap h^{-1}(U)$. Now it is easy to conclude that, since $Y_{0} \cap h^{-1}(U)$ is dense in $h^{-1}(U),(T f)^{\beta_{0} Y}(y)=0$ for every $y \in h^{-1}(U)$.

Lemma 2.5. Suppose that $T: C^{*}(X) \rightarrow C^{*}(Y)$ is an injective weakly separating map with closed range and one of the following statements holds:

- $T$ is additive and there exists a natural number $n \in \mathbb{K}$ such that $0<|n|<1$.

- $T$ is linear.

Let $x_{0} \in \beta_{0} X, y_{0} \in \beta_{0} Y_{0}$ be such that $h\left(y_{0}\right)=x_{0}$. If $f \in C^{*}(X)$ satisfies $f^{\beta_{0} X}\left(x_{0}\right)=0$, then $(T f)^{\beta_{0} Y_{0}}\left(y_{0}\right)=0$.

Proof. We shall give the proof when there exists a natural number in $\mathbb{K}$ with absolute value different from 0 and 1 . The case when $T$ is linear is similar. If $f^{\beta_{0} X}$ is equal to zero on a neighborhood of $x_{0}$ in $\beta_{0} X$, the result follows from Lemma 2.4. So we suppose that this is not the case. Assume that we can find $f \in C^{*}(X)$ such that $f^{\beta_{0} X}\left(x_{0}\right)=0$ and $(T f)^{\beta_{0} Y_{0}}\left(y_{0}\right) \neq 0$. Then consider a sequence of natural numbers $\left(\alpha_{n}\right)$ in $\mathbb{K}$ such that $\left(\left|\alpha_{n}\right|\right)$ is a strictly decreasing sequence in $\mathbb{R}$ converging to zero and such that $\lim _{n \rightarrow \infty}\left|\alpha_{n} / \alpha_{n+1}\right|=+\infty$. Define, for each $n \in \mathbb{N}$,

$$
U_{n}:=\left\{x \in X:|f(x)| \in\left[\left|\alpha_{n+1}\right|,\left|\alpha_{n}\right|\right)\right\} .
$$

and

$$
U_{0}:=\left\{x \in X:|f(x)| \geq\left|\alpha_{1}\right|\right\} .
$$

Clearly, every $U_{n}$ is clopen, so the products $f \xi_{U_{n}}$ belong to $C^{*}(X)$ for every $n \in \mathbb{N} \cup\{0\}$. We also have $f=\sum_{n=0}^{\infty} f \xi_{U_{n}}$. 
Now, given $n_{0} \in \mathbb{N} \cup\{0\}$, we have that $c\left(f \xi_{U_{n_{0}}}\right) \cap c\left(\sum_{n \neq n_{0}} f \xi_{U_{n}}\right)=\emptyset$ so $c\left(T\left(f \xi_{U_{n_{0}}}\right)\right) \cap c\left(T\left(\sum_{n \neq n_{0}} f \xi_{U_{n}}\right)\right)=\emptyset$, and in the same way $c\left(T\left(f \xi_{U_{n}}\right)\right) \cap c\left(T\left(f \xi_{U_{m}}\right)\right)$ $=\emptyset$ if $n \neq m$.

If the sequence $\left(\left\|T\left(f \xi_{U_{n}}\right)\right\|\right)$ converges to zero, then $\sum_{n=0}^{\infty} T\left(f \xi_{U_{n}}\right)$ converges in $C^{*}(Y)$. Assume the sequence is not convergent so there are a sequence $\left(y_{n}\right)$ in $Y$ and $\delta>0$ such that $\left|\left(T\left(f \xi_{U_{n}}\right)\right)\left(y_{n}\right)\right|>\delta$, for infinitely many $n \in \mathbb{N}$.

Now, defining $\alpha_{-1}=1=\alpha_{0}$, consider

$$
g:=\sum_{n=0}^{\infty} \frac{\alpha_{n-1}}{\alpha_{n}} f \xi_{U_{n}}
$$

We have that since $\left\|\left(\alpha_{n-1} / \alpha_{n}\right) f \xi_{U_{n}}\right\| \leq\left|\alpha_{n-1}\right|$ and $\left(\left|\alpha_{n}\right|\right)$ converges to zero, then $g \in C^{*}(X)$. Also, since $\alpha_{n}$ is a rational number for every $n$ and $T$ is additive and weakly separating, then by Lemma 2.4

$$
\left|(T g)\left(y_{n}\right)\right|=\left|\frac{\alpha_{n-1}}{\alpha_{n}}\right|\left|(T f)\left(y_{n}\right)\right| .
$$

This implies that $T g$ is not bounded, which is absurd.

Then we deduce that $\left(\left\|T\left(f \xi_{U_{n}}\right)\right\|\right)$ converges to zero, and consequently $\sum_{n=0}^{\infty} T\left(f \xi_{U_{n}}\right)$ is continuous. Moreover, as $T$ has closed range,

$$
f^{\prime}:=T f-\sum_{n=0}^{\infty} T\left(f \xi_{U_{n}}\right)
$$

is in the range of $T$, so there is $f_{0} \in C^{*}(X)$ such that $T f_{0}=f^{\prime}$.

Suppose that $f^{\prime} \neq 0$. Then $f_{0} \neq 0$. If $c\left(f_{0}\right) \cap c(f) \neq \emptyset$, consider $n \in \mathbb{N} \cup$ $\{0\}$ such that $U_{n} \cap c\left(f_{0}\right) \neq \emptyset$ and define $U:=U_{n}$. If $c\left(f_{0}\right) \cap c(f)=\emptyset$, take $U$ a nonempty clopen subset of $c\left(f_{0}\right)$ and take any $n \in \mathbb{N}$. Then, in any case, we have that $f_{0}=f_{0} \xi_{U}+f_{0} \xi_{X-U}$, where $c\left(T\left(f_{0} \xi_{U}\right)\right) \cap c\left(T\left(f_{0} \xi_{X-U}\right)\right)=\emptyset$, by the weakly separating property of $T$. Also, for the same reason, we have that $c\left(T\left(f_{0} \xi_{U}\right)\right) \cap c\left(T\left(\sum_{k \neq n} f \xi_{U_{k}}\right)\right)=\emptyset$, this is, $c\left(T\left(f_{0} \xi_{U}\right)\right) \cap c\left(\sum_{k \neq n} T\left(f \xi_{U_{k}}\right)+f^{\prime}\right)=\emptyset$. In particular, this means that $c\left(T\left(f_{0} \xi_{U}\right)\right) \cap c\left(f^{\prime}\right)=\emptyset$, which contradicts the equality $f^{\prime}=T\left(f_{0} \xi_{U}\right)+T\left(f_{0} \xi_{X-U}\right)$ and the fact that these last functions have disjoint cozero sets. So we have proved that $f^{\prime}=0$, this is, $T f=\sum_{n=0}^{\infty} T\left(f \xi_{U_{n}}\right)$.

We see that for any $n \in \mathbb{N}, x_{0} \notin \operatorname{cl}_{\beta_{0} X} U_{n}$, which by Lemma 2.4 implies that $\left(T\left(f \xi_{U_{n}}\right)\right)^{\beta_{0} Y_{0}}\left(y_{0}\right)=0$ for each $n \in \mathbb{N}$. Therefore, since $\left(\left\|T\left(f \xi_{U_{n}}\right)\right\|\right)$ converges to zero, $(T f)^{\beta_{0} Y_{0}}\left(y_{0}\right)=0$ and we are done.

Theorem 2.6. Suppose that $T: C^{*}(X) \rightarrow C^{*}(Y)$ is a weakly biseparating additive map. Then $h$ is a homeomorphism from $\beta_{0} Y$ onto $\beta_{0} X$.

Proof. Since $T^{-1}$ is additive, weakly biseparating and bijective, we can construct a continuous map $k: \beta_{0} X \rightarrow \beta_{0} Y$ in a similar way as we constructed $h$. In particular, we know that, given $f \in C^{*}(X)$ and $x \in \beta_{0} X$, if $f^{\beta_{0} X}(h(k(x))=0$, then $(T f)^{\beta_{0} Y}(k(x))=0$. Also, since $k(x)$ is the support point of $x$ (for $T^{-1}$ ), $f^{\beta_{0} X}(x)=0$, by Lemma 2.5. If $x$ and $h(k(x))$ were two different points of $\beta_{0} X$, then taking $f \in C^{*}(X)$ satisfying $f^{\beta_{0} X}(h(k(x)))=0$ and $f^{\beta_{0}}(x)=1$, we would arrive at a contradiction. Then for every $x \in \beta_{0} X, h(k(x))=x$ and, in the same way, we can prove that $k(h(y))=y$ for every $y \in \beta_{0} Y$. We can conclude that $h$ is bijective and its inverse is the continuous function $k$. 
Lemma 2.7. Suppose that $\alpha \in \mathbb{K}$ satisfies $0<|\alpha|<1$. Then given any $\beta \in \mathbb{K}$, $|\beta|>1$, there exists $n \in \mathbb{N}$ such that $|\alpha| \leq\left|\alpha^{n} \beta\right| \leq 1$.

Proof. It is clear that there exists $n_{0} \in \mathbb{N} \cup\{0\}$ such that $|\alpha|^{-n_{0}} \leq|\beta|<|\alpha|^{-\left(n_{0}+1\right)}$. Then it is easy to see that $n:=n_{0}+1 \in \mathbb{N}$ does the job.

Theorem 2.8. Suppose that $\mathbb{K}$ is not locally compact and has characteristic zero. Suppose that $T: C^{*}(X) \rightarrow C^{*}(Y)$ is a weakly biseparating additive map. Then $h$ is a homeomorphism from $Y$ onto $X$.

Proof. Suppose that $h\left(y_{0}\right) \in \beta_{0} X-X$ for some $y_{0} \in Y$. Then there exists a sequence $\left(U_{n}\right)$ of clopen neighborhoods of $h\left(y_{0}\right)$ in $\beta_{0} X$ such that $U_{n+1}$ is strictly contained in $U_{n}$ for every $n \in \mathbb{N}, U_{1}=\beta_{0} X$, and $X \cap \bigcap_{n=1}^{\infty} U_{n}=\emptyset$. Define $V_{n}:=U_{n}-U_{n+1}$ for every $n \in \mathbb{N}$.

Now we split the proof into two cases.

Case 1. Assume that there exists $n_{0} \in \mathbb{N}$ such that $\left|n_{0}\right| \neq 1$. Since $\mathbb{K}$ is not locally compact, there exists a sequence $\left(\alpha_{n}\right)$ in $\mathbb{K}$ such that $1-1 /(n+1) \leq\left|\alpha_{n}\right| \leq 1$ for every $n \in \mathbb{N}$, and $\left|\alpha_{n}-\alpha_{m}\right| \geq 1-1 /(n+1)$ for every $n, m \in \mathbb{N}, n>m$. Since $h: \beta_{0} Y \rightarrow \beta_{0} X$ is a homeomorphism, we have that, for each $n \in \mathbb{N}, \alpha_{n} \xi_{Y \cap h^{-1}\left(V_{n}\right)}$ belongs to $C^{*}(Y)$. Suppose that the sequence $\left(T^{-1}\left(\alpha_{n} \xi_{Y \cap h^{-1}\left(V_{n}\right)}\right)\right)$ is not bounded in $C^{*}(X)$. This implies that there exist a sequence $\left(n_{k}\right)$ in $\mathbb{N}$ with $n_{k} \neq n_{k^{\prime}}$ whenever $k \neq k^{\prime}$, and a sequence $\left(x_{k}\right)$ in $X$, such that each $x_{k}$ belongs to $V_{n_{k}}$ and $\left|\left(T^{-1}\left(\alpha_{n_{k}} \xi_{Y \cap h^{-1}\left(V_{n_{k}}\right)}\right)\right)\left(x_{k}\right)\right|>\left\|T^{-1}\left(\alpha_{n_{k}} \xi_{Y \cap h^{-1}\left(V_{n_{k}}\right)}\right)\right\|-1>k$, for every $k \in \mathbb{N}$. By Lemma 2.7 and for every $n_{k}$, there exists $m_{k} \in \mathbb{N}$ such $\beta_{k}:=n_{0}{ }^{m_{k}} \in \mathbb{Q}$ satisfies

$$
\left|n_{0}\right| \leq\left|\beta_{k}\right|\left|\left(T^{-1}\left(\alpha_{n_{k}} \xi_{Y \cap h^{-1}\left(V_{n_{k}}\right)}\right)\right)\left(x_{k}\right)\right| \leq 1
$$

On the other hand, it is easy to check that $f_{0}:=\sum_{k=1}^{\infty} \beta_{k} T^{-1}\left(\alpha_{n_{k}} \xi_{Y \cap h^{-1}\left(V_{n_{k}}\right)}\right)$ is a continuous bounded function on $X$. Also there exists an accumulation point $x_{0}$ of $\left(x_{k}\right)$ in $\beta_{0} X-X$. Since $\left|n_{0}\right| \leq\left|\beta_{k}\right|\left|\left(T^{-1}\left(\alpha_{n_{k}} \xi_{Y \cap h^{-1}\left(V_{n_{k}}\right)}\right)\right)\left(x_{k}\right)\right| \leq 1$ for every $k \in \mathbb{N}$, it is clear that $f_{0}^{\beta_{0} X}\left(x_{0}\right) \neq 0$, and by Lemma $2.5\left(T f_{0}\right)^{\beta_{0} Y_{0}}\left(h^{-1}\left(x_{0}\right)\right) \neq 0$. Also, since $h: \beta_{0} Y \rightarrow \beta_{0} X$ is a homeomorphism and $x_{0} \notin \bigcup_{k=1}^{\infty} V_{n_{k}}, h^{-1}\left(x_{0}\right)$ does not belong to $\bigcup_{k=1}^{\infty} h^{-1}\left(V_{n_{k}}\right)$. Also, using Lemma 2.4 it is not difficult to prove that, since $T$ is $\mathbb{Q}$-linear, $T f_{0}=\sum_{k=1}^{\infty} \beta_{k} \alpha_{n_{k}} \xi_{Y \cap h^{-1}\left(V_{n_{k}}\right)}$. Now, since $\lim _{k \rightarrow \infty} \beta_{k}=0$, it is easy to see that $\left(T f_{0}\right)^{\beta_{0} Y}(y)=0$ for every $y \notin \bigcup_{k=1}^{\infty} h^{-1}\left(V_{n_{k}}\right)$. Consequently $\left(T f_{0}\right)^{\beta_{0} Y_{0}}\left(h^{-1}\left(x_{0}\right)\right)=0$, which is a contradiction.

Then we have that $\left(T^{-1}\left(\alpha_{n} \xi_{Y \cap h^{-1}\left(V_{n}\right)}\right)\right)$ is a bounded sequence in $C^{*}(X)$, and consequently $f_{1}:=\sum_{n=1}^{\infty} T^{-1}\left(\alpha_{n} \xi_{Y \cap h^{-1}\left(V_{n}\right)}\right)$ belongs to $C^{*}(X)$. On the other hand, as above, it is easy to see that $T f_{1}=\sum_{n=1}^{\infty} \alpha_{n} \xi_{Y \cap h^{-1}\left(V_{n}\right)}$, this is, $1 / 2 \leq$ $\left|\left(T f_{1}\right)(y)\right| \leq 1$ for every $y \in Y$. Thus $\left(T f_{1}\right)\left(y_{0}\right)=\alpha \neq 0$. Now take a neighborhood $U$ of $y_{0}$ in $Y$ such that for every $y \in U,\left|\left(T f_{1}\right)(y)-\left(T f_{1}\right)\left(y_{0}\right)\right|<|\alpha| / 2$. Then there exist $n_{1}$ and $n_{2}$ in $\mathbb{N}, n_{1} \neq n_{2}$, and $y_{1}, y_{2}$ in $U$ such that $y_{1} \in h^{-1}\left(V_{n_{1}}\right)$ and $y_{2} \in h^{-1}\left(V_{n_{2}}\right)$. Consequently $\left(T f_{1}\right)\left(y_{1}\right)=\alpha_{n_{1}}$ and $\left(T f_{1}\right)\left(y_{2}\right)=\alpha_{n_{2}}$. But then we deduce that $\left|\left(T f_{1}\right)\left(y_{1}\right)-\left(T f_{1}\right)\left(y_{2}\right)\right|=\left|\alpha_{n_{1}}-\alpha_{n_{2}}\right|=|\alpha|$, which is not possible.

Therefore in this case $h(y) \in X$ for every $y \in Y$.

Case 2. Assume that $|n|=1$ for every $n \in \mathbb{N}$. Then take $f^{\prime}:=T^{-1} \mathbf{1}$ and $f_{2}:=\sum_{n=1}^{\infty} n \xi_{X \cap V_{n}} f^{\prime}$. Moreover, using Lemma 2.4, it is easy to see that $T f_{2}=$ $\sum_{n=1}^{\infty} n T\left(\xi_{X \cap V_{n}} f^{\prime}\right)=\sum_{n=1}^{\infty} n \xi_{Y \cap h^{-1}\left(V_{n}\right)}$. Since $h$ is a homeomorphism, given 
any open neighborhood $V$ of $y_{0}$ in $Y$, there are infinitely many $n \in \mathbb{N}$ such that $V \cap h^{-1}\left(V_{n}\right) \neq \emptyset$. This implies that every neighborhood of $y_{0}$ in $Y$ contains points at which $T f_{2}$ takes different values $n \in \mathbb{N}$, which is impossible because $T f_{2}$ is continuous and $|n|=1$ for every $n \in \mathbb{N}$.

This implies that in both cases $h(y)$ belongs to $X$ for every $y \in Y$, and in the same way $h^{-1}(x) \in Y$ for every $x \in X$, this is, $h: Y \rightarrow X$ is a surjective homeomorphism.

Remarks. 1. Note that Theorem 2.8 also holds for any $\mathbb{K}$ not locally compact (not necessarily of characteristic zero) when $T$ is linear. A slight modification of the proof of Case 2 shows it. The same remark also applies to Theorem 2.6 for any $\mathbb{K}$.

2. Given a separating additive and bijective map $T: C^{*}(X) \rightarrow C^{*}(Y)$, we cannot assure that $T$ is (weakly) biseparating nor that $X$ and $Y$ are homeomorphic, as the following example shows. Consider $X:=\{x\}, Y=\left\{y, y^{\prime}\right\}$ and $\mathbb{K}$ to be the field $\mathbb{C}_{p}$, for some prime $p$. Obviously every map $T: C^{*}(X) \rightarrow C^{*}(Y)$ is separating, but $X$ and $Y$ are not homeomorphic. Take bases $\mathcal{B}$ and $\mathcal{B}^{\prime}$ of $C^{*}(X)$ and $C^{*}(Y)$, respectively, regarded as linear spaces over the field $\mathbb{Q}$ of rational numbers. It is easy to check that $\mathcal{B}$ and $\mathcal{B}^{\prime}$ have the same cardinality, this is, there exists a bijective map $T: \mathcal{B} \rightarrow \mathcal{B}^{\prime}$. If we extend $T$ by $\mathbb{Q}$-linearity to the whole $C^{*}(X)$, we obtain the desired map.

\section{Automatic continuity Results}

Theorem 3.1. If $T: C^{*}(X) \rightarrow C^{*}(Y)$ is a bijective weakly separating linear map, then it is biseparating.

Proof. Given any $x \in X$, there exists $y \in \beta_{0} Y$ such that $h(y)=x$. Also, since $T$ is bijective, there exists $f_{0} \in C^{*}(X)$ such that $T f_{0}=\mathbf{1} \in C^{*}(Y)$. By Lemma 2.5, $f_{0}(x) \neq 0$. Now consider $f \in C^{*}(X)$ such that $f(x) \neq 0$. Clearly, there exists $\alpha \in \mathbb{K}, \alpha \neq 0$, such that $\left(f_{0}+\alpha f\right)(x)=0$, and by Lemma $2.5,(T f)^{\beta_{0} Y}(y) \neq 0$. In the same way, if $g \in C^{*}(X)$ satisfies $g(x) \neq 0,(T g)^{\beta_{0} Y}(y) \neq 0$. This implies that if $c(f) \cap c(g) \neq \emptyset$, then $c(T f) \cap c(T g) \neq \emptyset$, and then it is easy to conclude that $T^{-1}$ is separating. For the same reason, $T$ is also separating.

Theorem 3.2. Suppose that $\mathbb{K}$ is not locally compact, and that $T: C^{*}(X) \rightarrow$ $C^{*}(Y)$ is a bijective weakly separating linear map. Then $h$ is a homeomorphism from $Y$ onto $X$ and there exists a continuous map $a: Y \rightarrow \mathbb{K}$ such that for every $y \in Y$ and $f \in C^{*}(X)$,

$$
(T f)(y)=a(y) f(h(y)) .
$$

Moreover $\inf _{y \in Y}|a(y)|>0$ and $T$ is continuous.

Proof. By Remark 1 after Theorem 2.8, $h: Y \rightarrow X$ is a homeomorphism. Define $a:=T$ 1. By Theorem 3.1, $T$ is biseparating and consequently by Lemma $2.5, a$ does not vanish at any point of $Y$. On the other hand, given $y \in Y$ and $f \in C^{*}(X)$, then we can define $g \in C^{*}(X)$ as the function constantly equal to $f(h(y))$ on $X$. It is clear that $(f-g)(h(y))=0$ and by Lemma 2.5, $(T f)(y)=(T g)(y)=f(h(y))(T \mathbf{1})(y)$, this is, $(T f)(y)=a(y) f(h(y))$.

Also, if there exists a sequence $\left(y_{n}\right)$ in $Y$ with $\lim _{n \rightarrow \infty} a\left(y_{n}\right)=0$, it is easy to see that $\left(\left|\left(T^{-1} \mathbf{1}\right)\left(h\left(y_{n}\right)\right)\right|\right)$ converges to $\infty$, which is not possible. Consequently $\inf _{y \in Y}|a(y)|>0$. 
Finally, we are going to see that $T$ is bounded. Given $f \in C^{*}(X)$, we have

$$
\|T f\|=\sup _{y \in Y}|a(y) f(h(y))| \leq \sup _{y \in Y}|a(y)|\|f\| .
$$

Taking into account that $a$ is bounded, the result follows.

Now the proof of the following result is easy.

Corollary 3.3. Suppose that $\mathbb{K}$ is not locally compact, and that $T: C^{*}(X) \rightarrow$ $C^{*}(Y)$ is an algebra isomorphism. Then there exists a surjective homeomorphism $h: Y \rightarrow X$ such that $T f=f \circ h$ for every $f \in C(X)$.

Definition 3.1. A function $f: X \rightarrow \mathbb{K}$ is said to be nonvanishing if $f(x) \neq 0$ for every $x \in X$.

Definition 3.2. $T: C^{*}(X) \rightarrow C^{*}(Y)$ is said to be nonvanishing if it maps nonvanishing functions into nonvanishing functions.

Theorem 3.4. Suppose that $\mathbb{K}$ is not locally compact. If a bijective linear map $T: C^{*}(X) \rightarrow C^{*}(Y)$ is nonvanishing, then it is biseparating and continuous.

Proof. Take $f \in C^{*}(X)$ nonvanishing and a clopen subset $U$ of $X$. We are going to prove that $c\left(T\left(\xi_{U} f\right)\right) \cap c\left(T\left(\xi_{X-U} f\right)\right)=\emptyset$. Otherwise, take $y \in Y$ such that $\left(T\left(\xi_{U} f\right)\right)(y)=\alpha,\left(T\left(\xi_{X-U} f\right)\right)(y)=\beta$, and $\alpha \beta \neq 0$. It is clear that if we take $\gamma=$ $-\beta / \alpha$, then $\gamma \xi_{U} f+\xi_{X-U} f$ is nonvanishing, whereas $\left(T\left(\gamma \xi_{U} f+\xi_{X-U} f\right)\right)(y)=0$, which is a contradiction.

Next we are going to see that $T$ is weakly separating and, by Theorem 3.1, it will be biseparating. Suppose that $f, g \in C^{*}(X)$ are such that there exists a clopen subset $U$ of $X$ satisfying $c(f) \subset U$ and $c(g) \subset X-U$. Take $f_{0} \in C^{*}(X)$ nonvanishing and such that $\left|f_{0}(x)\right|>|f(x)|,|g(x)|$ for every $x \in X$. Suppose that $y \in c(T f) \cap c(T g)$. Then either $\left(T\left(\xi_{U} f_{0}\right)\right)(y)=0$ or $\left(T\left(\xi_{X-U} f_{0}\right)\right)(y)=0$. Without loss of generality we can assume that $\left(T\left(\xi_{U} f_{0}\right)\right)(y)=0$. Consequently $\left(T\left(\xi_{X-U} f_{0}\right)\right)(y) \neq 0$. Consider $\gamma \in \mathbb{K}-\{0\}$ such that

$$
\gamma(T f)(y)+\left(T\left(\xi_{X-U} f_{0}\right)\right)(y)=0 .
$$

Then $\gamma f+\gamma \xi_{U} f_{0}+\xi_{X-U} f_{0}$ is nonvanishing, although $\left(T\left(\gamma f+\gamma \xi_{U} f_{0}+\xi_{X-U} f_{0}\right)\right)(y)=$ 0 , which is a contradiction. We conclude that $T$ is biseparating, and by Theorem 3.2, it is continuous.

The author wishes to thank the referee for making several suggestions which improved this paper.

\section{REFERENCES}

[A] J. Araujo, Nonarchimedean Šilov boundaries and multiplicative isometries, Indag. Math., N. S. 8 (1997), 417-431. CMP 98:12

[ABN1] J. Araujo, E. Beckenstein and L. Narici, Biseparating maps and homeomorphic realcompactifications, J. Math. Anal. App. 192 (1995), 258-265. MR 96b:46038

[ABN2] J. Araujo, E. Beckenstein and L. Narici, Separating maps and the nonarchimedean Hewitt theorem, Ann. Math. Blaise Pascal 2 (1995), 19-27. MR 96d:46099

[ABN3] J. Araujo, E. Beckenstein and L. Narici, When is a separating map biseparating?, Archiv Math. 67 (1996), 395-407. MR 97f:47026

[AF] J. Araujo and J. J. Font, Linear isometries between subspaces of continuous functions, Trans. A.M.S. 349 (1997), 413-428. MR 97d:46026

[BNT] E. Beckenstein, L. Narici and A. R. Todd, Automatic continuity of linear maps on spaces of continuous functions, Manuscripta Math. 62 (1988), 257-275. MR 89j:47020 
[GJ] L. Gillman and M. Jerison, Rings of continuous functions. Van Nostrand, Princeton, 1960. MR 22:6994

[H] M. Henriksen, On the equivalence of the ring, lattice, and semigroup of continuous functions, Proc. Amer. Math. Soc. 7 (1956), 959-960. MR 18:559a

[J] K. Jarosz, Automatic continuity of separating linear isomorphisms, Canad. Math. Bull. 33 (1990), 139-144. MR 92j:46049

[vP] M. van der Put, Algèbres de fonctions continues p-adiques, Indag. Math. 30 (1968), 401-420. MR 39:784

[vR] A. C. M. van Rooij, Nonarchimedean Functional Analysis, Dekker, New York 1978. MR 81a:46084

[S] R. Staum, The algebra of bounded continuous functions into a nonarchimedean field, Pacific J. Math. 50 (1974), 169-185. MR 49:5803

Departamento de Matemáticas, Estadística y Computación, Universidad de Cantabria, Facultad de Ciencias, Avda. de los Castros, s. N., E-39071 Santander, Spain

E-mail address: araujo@matesco.unican.es 